\title{
Comments on the Chernoff estimate
}

\author{
Valentin A. Zagrebnov \\ Institut de Mathématiques de Marseille, 13453 Marseille, France \\ Valentin.Zagrebnov@univ-amu.fr
}

PACS 02.30.Sa, 02.30.Tb

ABSTRACT The Chernoff $\sqrt{n}$-Lemma is revised. This concerns two aspects: a re-examination of the Chernoff estimate in the strong operator topology and the operator-norm estimate for quasi-sectorial contractions. Applications to the Lie-Trotter product formula approximation $C_{0}$-semigroups are also discussed.

KEYWORDS Chernoff lemma, Semigroup theory, Product formula, Convergence rate.

ACKNOWLEDGEMENTS The strong convergence with the rate (c) (see, Section 3) was established for the first time by T. Möbus and C. Rouzé in [1, Lemma 4.2] by the method, which is different to that in our Lemma 3.1. I am thankful to Tim Möbus for useful correspondences and, in particular, for attracting my attention to an inconsistency in [2, Lemma 2.1], which is corrected in the present paper. These Comments are based on my lecture delivered on the Pierre Duclos Workshop (20-22 September 2021) at National Research University ITMO, Russian Federation. I am grateful to Prof. Igor Yu. Popov for invitation.

FOR CITATION Zagrebnov V.A. Comments on the Chernoff estimate. Nanosystems: Phys. Chem. Math., 2022, 13 (1), 17-23.

\section{Introduction}

Recall that the Chernoff $\sqrt{n}$-Lemma [3, Lemma 2] is one of a key tool in the theory of semigroup approximations, see e.g. [4, Chapter III, Section 5]. For the reader's convenience and for motivation of the present comments, we show this lemma below.

Lemma 1.1. Let bounded operator $C$ on a Banach space $\mathfrak{X}(C \in \mathcal{L}(\mathfrak{X}))$ be a contraction, i.e., $\|C\| \leq 1$. Then, $\left\{e^{t(C-1)}\right\}_{t \geq 0}$ is a norm-continuous contraction semigroup on $\mathfrak{X}$ and one has the estimate:

$$
\left\|\left(C^{n}-e^{n(C-1)}\right) x\right\| \leq \sqrt{n}\|(C-\mathbb{1}) x\|,
$$

for all $x \in \mathfrak{X}$ and $n \in \mathbb{N}$.

Proof. To prove the inequality (1.1) we use the representation:

$$
C^{n}-e^{n(C-1)}=e^{-n} \sum_{m=0}^{\infty} \frac{n^{m}}{m !}\left(C^{n}-C^{m}\right) .
$$

To proceed we insert:

$$
\left\|\left(C^{n}-C^{m}\right) x\right\| \leq\left\|\left(C^{|n-m|}-\mathbb{1}\right) x\right\| \leq|m-n|\|(C-\mathbb{1}) x\|,
$$

into (1.2) to obtain by the Cauchy-Schwarz inequality the estimate:

$$
\begin{aligned}
& \left\|\left(C^{n}-e^{n(C-1)}\right) x\right\| \leq\|(C-\mathbb{1}) x\| e^{-n} \sum_{m=0}^{\infty} \frac{n^{m}}{m !}|m-n| \leq \\
& \left\{\sum_{m=0}^{\infty} e^{-n} \frac{n^{m}}{m !}|m-n|^{2}\right\}^{1 / 2}\|(C-\mathbb{1}) x\| . x \in \mathfrak{X},
\end{aligned}
$$

Note that the sum in the right-hand side of (1.4) can be calculated explicitly. This gives the value $n$, which yields (1.1).

The aim of the present comments is to revise the Chernoff $\sqrt{n}$-Lemma in two directions. First, we modify the $\sqrt{n}$ estimate (1.1) for contractions. Then, we apply two new estimates for the proof of the Chernoff product formula for strongly continuous semigroups $\left(C_{0}\right.$-semigroups) in the strong operator topology, see Section 2 and Section 3.

Second, we use the idea of the probabilistic approach to the estimate in strong operator topology (Section 2) to uplift it to the operator-norm estimate for a special class of contractions: the quasi-sectorial contractions, see Section 4. 


\section{Revised $\sqrt{n}$-Lemma and Chernoff product formula}

We start by a technical lemma. It is a revised version of the Chernoff $\sqrt{n}$-Lemma 1.1. Our variational estimate (2.1) in $\sqrt[3]{n}$-Lemma 2.1 and the probabilistic approach are, in a certain sense, more flexible than (1.1). Indeed, the scheme of the proof will be used later (Section 4) for uplifting the convergence of the Chernoff and the Lie-Trotter product formulae to the operator-norm topology.

Lemma 2.1. ( $\sqrt[3]{n}$-Lemma) Let $C$ be a contraction on a Banach space $\mathfrak{X}$. Then, $\left\{e^{t(C-1}\right\}_{t \geq 0}$ is a norm-continuous contraction semigroup on $\mathfrak{X}$ and one has the estimate:

$$
\left\|\left(C^{n}-e^{n(C-1)}\right) x\right\| \leq \frac{n}{\epsilon_{n}^{2}} 2\|x\|+\epsilon_{n}\|(\mathbb{1}-C) x\|, \quad n \in \mathbb{N} \backslash\{0\},
$$

for all $x \in \mathfrak{X}$ and $\epsilon_{n}>0$. For the optimal value of the parameter $\epsilon_{n}:$

$$
\epsilon_{n}^{*}:=\left(\frac{4 n\|x\|}{\|(\mathbb{1}-C) x\|}\right)^{1 / 3},
$$

on the right-hand side of (2.1), we obtain the estimate:

$$
\left\|\left(C^{n}-e^{n(C-\mathbb{1})}\right)\right\| \leq \frac{3}{2} \sqrt[3]{n}\|2(\mathbb{1}-C)\|^{2 / 3},
$$

which is the $\sqrt[3]{n}$-Lemma.

Proof. Since operator $C$ is bounded and $\|C\| \leq 1$, the operator $(\mathbb{1}-C)$ is the generator of a norm-continuous contraction semigroup:

$$
\left\|e^{t(C-1)}\right\| \leq e^{-t}\left\|\sum_{m=0}^{\infty} \frac{t^{m}}{m !} C^{m}\right\| \leq 1 .
$$

In order to prove estimate (2.1), we use the representation:

$$
C^{n}-e^{n(C-1)}=e^{-n} \sum_{m=0}^{\infty} \frac{n^{m}}{m !}\left(C^{n}-C^{m}\right) .
$$

Then, we split the sum (2.5) into two parts: the central part for $|m-n| \leq \epsilon_{n}$ and the tails for $|m-n|>\epsilon_{n}$. Optimisation of the splitting parameter $\epsilon_{n}$ in (2.1) yields the best estimate and thus the optimal value of $\delta \in \mathbb{R}$.

For evaluation of the tails, we use the Tchebychëv inequality. Let $X_{n} \in \mathbb{N}_{0}$ be the Poisson random variable with the rate parameter $n$, that is, with the probability distribution $\mathbb{P}\left\{X_{n}=m\right\}=n^{m} e^{-n} / m !$. Then, one gets for the expectation: $\mathbb{E}\left(X_{n}\right)=n$, and for the variance: $\operatorname{Var}\left(X_{n}\right):=\mathbb{E}\left(\left(X_{n}-\mathbb{E}\left(X_{n}\right)\right)^{2}\right)=n$. That being so, the Tchebychëv inequality yields

$$
\mathbb{P}\left\{\left|X_{n}-\mathbb{E}\left(X_{n}\right)\right|>\epsilon\right\} \leq \frac{\operatorname{Var}\left(X_{n}\right)}{\epsilon_{n}^{2}}, \text { for any } \epsilon_{n}>0 .
$$

Note that although for any $x \in \mathfrak{X}$ there is an evident bound: $\left\|\left(C^{n}-C^{m}\right) x\right\| \leq 2\|x\|$, for estimating (2.5) we shall also use below inequalities:

$$
\begin{aligned}
\left\|\left(C^{n}-C^{m}\right) x\right\| & =\left\|C^{n-k}\left(C^{k}-C^{m-n+k}\right) x\right\| \\
& \leq|m-n|\left\|C^{n-k}(\mathbb{1}-C) x\right\|, \quad k=0,1, \ldots, n .
\end{aligned}
$$

Then by $\|C\| \leq 1$ and by the Tchebychëv inequality (2.6) we obtain the estimate for tails:

$$
\begin{aligned}
& e^{-n} \sum_{|m-n|>\epsilon_{n}} \frac{n^{m}}{m !}\left\|\left(C^{n}-C^{m}\right) x\right\| \leq e^{-n} \sum_{|m-n|>\epsilon_{n}} \frac{n^{m}}{m !} \cdot 2\|x\| \\
& =\mathbb{P}\left\{\left|X_{n}-\mathbb{E}\left(X_{n}\right)\right|>\epsilon_{n}\right\} \cdot 2\|x\| \leq \frac{n}{\epsilon_{n}^{2}} 2\|x\| .
\end{aligned}
$$

To evaluate the central part of the sum (2.5), when $|m-n| \leq \epsilon_{n}$, note that by virtue of (2.7):

$$
\begin{aligned}
\left\|\left(C^{n}-C^{m}\right) x\right\| & \leq|m-n|\left\|C^{n-\left[\epsilon_{n}\right]}(\mathbb{1}-C) x\right\| \\
& \leq \epsilon_{n}\|(\mathbb{1}-C) x\| .
\end{aligned}
$$

Then we obtain:

$$
e^{-n} \sum_{|m-n| \leq \epsilon_{n}} \frac{n^{m}}{m !}\left\|\left(C^{n}-C^{m}\right) x\right\| \leq \epsilon_{n}\|(\mathbb{1}-C) x\|, \quad x \in \mathfrak{X},
$$

for $n \in \mathbb{N} \backslash\{0\}$. Estimate (2.10), together with (2.8), yield (2.1) for all $u \in \mathfrak{X}$ and $\epsilon_{n}>0$. 
Minimising the estimate (2.1) with respect to parameter $\epsilon_{n}>0$ one obtains the optimal value for $\epsilon_{n}^{*}(2.2)$ and

$$
\frac{n}{\epsilon_{n}^{* 2}} 2\|x\|+\epsilon_{n}^{*}\|(\mathbb{1}-C) x\|=\frac{3}{2} \sqrt[3]{n}(4\|x\|)^{1 / 3}\|(\mathbb{1}-C) x\|^{2 / 3},
$$

for all $x \in \mathfrak{X}$ and $n \in \mathbb{N} \backslash\{0\}$. As a consequence, (2.1) and (2.11) yield (2.3), which is the $\sqrt[3]{n}$-Lemma.

Theorem 2.2. (Chernoff product formula) Let $\Phi: t \mapsto \Phi(t)$ be a function from $\mathbb{R}_{0}^{+}$to contractions on $\mathfrak{X}$ such that $\Phi(0)=\mathbb{1}$. Let $\left\{U_{A}(t)\right\}_{t \geq 0}$ be a contraction $C_{0}$-semigroup, and let $D \subset \operatorname{dom}(A)$ be a core of the generator $A$. and if

If the function $\Phi(t)$ has a strong right-derivative $\Phi^{\prime}(+0)$ at $t=0$ (that is, $\Phi^{\prime}(+0)$ x exists for any $x \in \operatorname{dom}\left(\Phi^{\prime}(+0)\right)$ )

for all $x \in D$, then

$$
\Phi^{\prime}(+0) x:=\lim _{t \rightarrow+0} \frac{1}{t}(\Phi(t)-\mathbb{1}) x=-A x
$$

for all $t \in \mathbb{R}_{0}^{+}$and $x \in \mathfrak{X}$.

$$
\lim _{n \rightarrow \infty}[\Phi(t / n)]^{n} x=U_{A}(t) x
$$

Proof. Consider the bounded approximations $\left\{A_{n}(s)\right\}_{n \geq 1}$ of generator $A$ :

$$
A_{n}(s):=\frac{\mathbb{1}-\Phi(s / n)}{s / n}, \quad s \in \mathbb{R}^{+}, \quad n \in \mathbb{N} .
$$

Note that these operators are $m$-accretive: $\left\|\left(A_{n}(s)+\zeta \mathbb{1}\right)^{-1}\right\| \leq(\operatorname{Re}(\zeta))^{-1}$ for $\operatorname{Re}(\zeta)>0$ and for any $n \in \mathbb{N}$. By $\|\Phi(t)\| \leq 1$ together with (2.13) we obtain $\left\|e^{-t A_{n}(s)}\right\| \leq 1$, but also

$$
\lim _{n \rightarrow \infty} A_{n}(s) x=A x,
$$

for all $x \in D$ and any $s \in \mathbb{R}^{+}$. Then, given that $D=\operatorname{core}(A)$, by virtue of the Trotter-Neveu-Kato generalised strong convergence theorem (see, e.g., [5, Theorem 3.17] or [4, Chapter III, Theorem 4.8]) one obtains

$$
\lim _{n \rightarrow \infty} e^{-t A_{n}(s)} x=U_{A}(t) x, \quad x \in \mathfrak{X}, \quad t \in \mathbb{R}_{0}^{+} .
$$

This is the strong and uniform in $t$ and in $s$ convergence (2.15) of contractive approximants $\left\{e^{-t A_{n}(s)}\right\}_{n \geq 1}$ for $t \in[0, \tau]$ and $s \in\left(0, s_{0}\right]$.

Now, by Lemma 2.1 for contraction $C:=\Phi(t / n)$ we obtain owing to (2.3) that:

$$
\begin{aligned}
& \left\|[\Phi(t / n)]^{n} x-e^{-t A_{n}(t)} x\right\|=\left\|\left([\Phi(t / n)]^{n}-e^{n(\Phi(t / n)-\mathbb{1})}\right) x\right\| \\
& \leq \frac{3}{2} \sqrt[3]{n}(4\|x\|)^{1 / 3}\|(\mathbb{1}-\Phi(t / n)) x\|^{2 / 3}, \quad x \in \mathfrak{X} .
\end{aligned}
$$

Since by (2.14) one gets for any $x \in D$ and uniformly on $\left(0, t_{0}\right]$ :

$$
\lim _{n \rightarrow \infty} \sqrt[3]{n}\|(\mathbb{1}-\Phi(t / n)) x\|^{2 / 3}=\lim _{n \rightarrow \infty} t^{2 / 3} n^{-1 / 3}\left\|A_{n}(t) x\right\|^{2 / 3}=0,
$$

equations (2.16) and (2.17) provide uniformly on $\left(0, t_{0}\right]$ :

$$
\lim _{n \rightarrow \infty}\left\|[\Phi(t / n)]^{n} x-e^{-t A_{n}(t)} x\right\|=0, \quad x \in D .
$$

Then, (2.15) and (2.18) yield uniformly in $t \in\left[0, t_{0}\right]$ limit:

$$
\lim _{n \rightarrow \infty}[\Phi(t / n)]^{n} x=U_{A}(t) x, \quad x \in D .
$$

Note that by density of $D$ and by the uniform estimate $\left\|[\Phi(t / n)]^{n} x-e^{-t A_{n}(t)} x\right\| \leq 2\|x\|$ the convergence in (2.18) can be extended to all $x \in \mathfrak{X}$. Indeed, it is known that on the bounded subsets of $\mathcal{L}(\mathfrak{X})$ the topology of point-wise convergence on a dense subset $D \subset \mathfrak{X}$ coincides with the strong operator topology, see, e.g., [6, Chapter III, Lemma 3.5]. As a consequence, limit (2.18) being extended to $x \in \mathfrak{X}$ and limit (2.15) yield (2.12).

The limit (2.12) is called the Chernoff product formula in the strong operator topology for contractive $C_{0}$-semigroup $\left\{U_{A}(t)\right\}_{t \geq 0}$.

Proposition 2.3. [3] (Lie-Trotter product formula) Let $A, B$ and $C$ be generators of contraction $C_{0}$-semigroups on $\mathfrak{X}$. Suppose that algebraic sum:

$$
C x=A x+B x,
$$

is valid for all $x \in D$, where domain $D=$ core $(C)$. Then, the semigroup $\left\{U_{C}(t)\right\}_{t \geq 0}$ can be approximated on $\mathfrak{X}$ in the strong operator topology by the Lie-Trotter product formula:

$$
e^{-t C} x=\lim _{n \rightarrow \infty}\left(e^{-t A / n} e^{-t B / n}\right)^{n} x, \quad x \in \mathfrak{X},
$$


for all $t \in \mathbb{R}_{0}^{+}$and $C:=\overline{(A+B)}$, which is closure of the sum (2.20).

Proof. Let us define the contraction $\mathbb{R}_{0}^{+} \ni t \mapsto \Phi(t), \Phi(0)=\mathbb{1}$, by:

$$
\Phi(t):=e^{-t A} e^{-t B} .
$$

Note that if $x \in D$, then derivative

$$
\Phi^{\prime}(+0) x=\lim _{t \rightarrow+0} \frac{1}{t}(\Phi(t)-\mathbb{1}) x=-(A+B) x .
$$

Now, we are in position to apply Theorem 2.2. This yields (2.21) for $C:=\overline{(A+B)}$.

Corollary 2.4. Extension of the strongly convergent Lie-Trotter product formula of Proposition 2.3 to quasi-bounded and holomorphic semigroups follows through verbatim.

\section{Revision of the Chernoff estimate}

In this section, we show a one more Chernoff-type estimate (3.1), which is of a different nature than the variational estimate (2.1) $(\sqrt[3]{n}$-Lemma 2.1). In fact, it is a kind of improvement of the original Chernoff estimate (1.1) $(\sqrt{n}$-Lemma $1.1)$.

Lemma 3.1. Let $C \in \mathcal{L}(\mathfrak{X})$ be contraction on a Banach space $\mathfrak{X}$. Then $\left\{e^{t(C-1}\right\}_{t \geq 0}$ is a norm-continuous contraction semigroup on $\mathfrak{X}$ and the following estimate:

$$
\left\|\left(C^{n}-e^{n(C-\mathbb{1})}\right) x\right\| \leq \frac{n}{2}\left(\left\|(C-\mathbb{1})^{2} x\right\|+\frac{e^{2}}{3}\left\|(C-\mathbb{1})^{3} x\right\|\right),
$$

holds for all $n \in \mathbb{N}$ and $x \in \mathfrak{X}$.

Proof. The first assertion is proven in Lemma 2.1, see (2.4).

To prove inequality (3.1) we use the telescopic representation:

$$
C^{n}-e^{n(C-1)}=\sum_{k=0}^{n-1} C^{n-k-1}\left(C-e^{(C-1)}\right) e^{k(C-1)} .
$$

To proceed we exploit that operator $C \in \mathcal{L}(\mathfrak{X})$ is bounded and therefore:

$$
C-e^{(C-\mathbb{1})}=-\frac{1}{2}(\mathbb{1}-C)^{2}-(\mathbb{1}-C)^{3} \sum_{m=3}^{\infty} \frac{(-1)^{m}}{m !}(\mathbb{1}-C)^{m-3},
$$

Owing to $\|C\| \leq 1$ one obtains the estimate:

$$
\left\|\sum_{m=3}^{\infty} \frac{1}{m !}(\mathbb{1}-C)^{m-3}\right\| \leq \frac{1}{6} e^{\|1-C\|} \leq \frac{e^{2}}{6} .
$$

Then on account of (3.2) - (3.4) and (2.4) we obtain inequality (3.1).

Corollary 3.2. (Chernoff product formula) Let $\Phi: t \mapsto \Phi(t)$ be a function from $\mathbb{R}_{0}^{+}$to contractions on $\mathfrak{X}$ such that $\Phi(0)=\mathbb{1}$, which satisfies conditions of Theorem 2.2. Then

$$
\lim _{n \rightarrow \infty}\left\|\left([\Phi(t / n)]^{n}-e^{n(\Phi(t / n)-1)}\right) x\right\|=0, \quad x \in \mathfrak{X},
$$

and one gets the product formula (2.12).

Proof. On account of (3.1) we obtain estimate

$$
\begin{aligned}
& \left\|\left([\Phi(t / n)]^{n}-e^{n(\Phi(t / n)-1)}\right) x\right\| \leq \\
& \frac{t^{2}}{2 n}\left(\left\|\frac{n^{2}}{t^{2}}(\mathbb{1}-\Phi(t / n))^{2} x\right\|+\frac{e^{2}}{3} \frac{t}{n}\left\|\frac{n^{3}}{t^{3}}(\mathbb{1}-\Phi(t / n))^{3} x\right\|\right), \quad x \in \mathfrak{X} .
\end{aligned}
$$

Note that by (2.14) we have on the dense set $D=\operatorname{core}(A)$ for any $t \in \mathbb{R}^{+}$:

$$
\lim _{n \rightarrow \infty} \frac{n}{t}(\mathbb{1}-\Phi(t / n)) x=A x, \quad x \in D .
$$

Given that generator $A$ of contractive $C_{0}$-semigroup is accretive, for $\operatorname{Re}(\zeta)>0$ the range of resolvent: $\operatorname{ran}((A+$ $\left.\zeta \mathbb{1})^{-1}\right)=\mathfrak{X}$. As a consequence (cf. [6, Chapter III, Problem 2.9]), domains $\operatorname{dom}\left(A^{2}\right) \supset \operatorname{dom}\left(A^{3}\right)$ are dense in $\mathfrak{X}$ and limit (3.7) provides:

$$
\lim _{n \rightarrow \infty}\left(A_{n}(t)\right)^{2} x=A^{2} x, \quad \lim _{n \rightarrow \infty}\left(A_{n}(t)\right)^{3} x=A^{3} x, \quad x \in D \subset \operatorname{dom}\left(A^{3}\right),
$$

where $A_{n}(t)=(t / n)^{-1}(\mathbb{1}-\Phi(t / n))$. 
By virtue of estimate (3.6) and (3.8), we obtain:

$$
\lim _{n \rightarrow \infty}\left\|\left([\Phi(t / n)]^{n}-e^{n(\Phi(t / n)-\mathbb{1})}\right) x\right\|=0, \quad x \in D .
$$

Then similarly to concluding arguments in Theorem 2.2 (that on the bounded subsets of $\mathcal{L}(\mathfrak{X})$ the topology of pointwise convergence on a dense subset $D \subset \mathfrak{X}$ coincides with the strong operator topology) the limit (3.9) can be extended to $x \in \mathfrak{X}$.

Now, given that $D=\operatorname{core}(A)$, by virtue of the Trotter-Neveu-Kato theorem we obtain the limit (2.15), and owing to (3.9) for $x \in \mathfrak{X}$, we deduce Chernoff product formula (2.12).

Resuming the Chernoff $\sqrt{n}$-inequality: (1.1), and its variety: (2.1) and (3.1), we conclude that due to the terms with $\|(C-\mathbb{1}) x\|$ all of them control only the strong convergence of the product formulae. The rates: $R_{n}(t)$, of these converges conditioned to $x \in D$ have the following asymptotic form for $t>0$ and large $n \in \mathbb{N}$ :

(a) For (1.1): $R_{n}(t)=1 / \sqrt{n}\left\|A_{n}(t) x\right\|$.

(b) For (2.1): $R_{n}(t)=1 / \sqrt[3]{n}\left\|A_{n}(t) x\right\|^{2 / 3}$.

(c) For (3.1): $R_{n}(t)=1 / n\left\|A_{n}(t)^{2} x\right\|$.

Remark 3.3. None of these three methods has an evident straightforward extension that could ensure the operator-norm convergence of the Chernoff product formula. In the next Section 4 we show that a relatively sophisticated method (cf.(b)) based on the Tchebychëv inequality (Section 2) is a fortiori more accurate to allow uplifting the convergence of the Chernoff product formula to the operator-norm topology for quasi-sectorial contractions on a Hilbert space.

\section{Quasi-sectorial contractions and $(\sqrt[3]{n})^{-1}$-Theorem}

Definition 4.1. [7] A contraction $C$ on the Hilbert space $\mathfrak{H}$ is called quasi-sectorial with semi-angle $\alpha \in[0, \pi / 2)$ with respect to the vertex at $z=1$, if its numerical range $W(C) \subseteq D_{\alpha}$. Here

$$
D_{\alpha}:=\{z \in \mathbb{C}:|z| \leq \sin \alpha\} \cup\{z \in \mathbb{C}:|\arg (1-z)| \leq \alpha \text { and }|z-1| \leq \cos \alpha\} .
$$

We comment that $D_{\alpha=\pi / 2}=\mathbb{D}$ (unit disc) and recall that a general contraction $C$ satisfies condition: $W(C) \subseteq \mathbb{D}$.

Note that if operator $C$ is a quasi-sectorial contraction, then $\mathbb{1}-C$ is an $m$-sectorial operator with vertex $z=0$ and semi-angle $\alpha$. Then for $C$ the limits: $\alpha=0$ and $\alpha=\pi / 2$, correspond respectively to self-adjoint and to standard contractions whereas for $\mathbb{1}-C$ they give a non-negative self-adjoint and an $m$-accretive (bounded) operators.

For $\lambda>0$ the resolvent $(A+\lambda \mathbb{1})^{-1}$ of an $m$-sectorial operator $A$, with semi-angle $\alpha \in\left[0, \alpha_{0}\right], \alpha_{0}<\pi / 2$, and vertex at $z=0$, gives an example of the quasi-sectorial contraction.

Proposition 4.2. [7,8] If $C$ is a quasi-sectorial contraction on a Hilbert space $\mathfrak{H}$ with semi-angle $0 \leq \alpha<\pi / 2$, then

$$
\left\|C^{n}(\mathbb{1}-C)\right\| \leq \frac{K_{\alpha}}{n+1}, n \in \mathbb{N} .
$$

The property (4.2) implies that the quasi-sectorial contractions belong to the class of so-called Ritt's operators [9]. This allows one to go beyond the $\sqrt[3]{n}$-Lemma 2.1 to the $(\sqrt[3]{n})^{-1}$-Theorem and from estimates in the strong operator topology to the operator-norm topology.

Theorem 4.3. $\left((\sqrt[3]{n})^{-1}\right.$-Theorem) Let $C$ be a quasi-sectorial contraction on $\mathfrak{H}$ with numerical range $W(C) \subseteq D_{\alpha}$, $0 \leq \alpha<\pi / 2$. Then

where $M_{\alpha}=2 K_{\alpha}+2$ and $K_{\alpha}$ is defined by (4.2).

$$
\left\|C^{n}-e^{n(C-1)}\right\| \leq \frac{M_{\alpha}}{n^{1 / 3}}, n \in \mathbb{N},
$$

Proof. With help of inequality (4.2) we can improve the estimate of the central part of the sum (2.5) in Lemma 2.1. Note that on account of (2.7) we obtain by (4.2) and $\|C\| \leq 1$ :

$$
\left\|C^{n}-C^{m}\right\| \leq|m-n|\left\|C^{n-\left[\epsilon_{n}\right]}(\mathbb{1}-C)\right\| \leq \epsilon_{n} \frac{K_{\alpha}}{n-\left[\epsilon_{n}\right]+1},
$$

cf. (2.9). Here $\epsilon_{n}:=n^{\delta+1 / 2}$ for $\delta<1 / 2$, which makes sense for the estimate (2.8) of tails, and $\left[\epsilon_{n}\right]$ is the integer part of $\epsilon_{n} \geq|m-n|$. Then owing to (4.4) the central part has the estimate:

$$
e^{-n} \sum_{|m-n| \leq \epsilon_{n}} \frac{n^{m}}{m !}\left\|\left(C^{n}-C^{m}\right) x\right\| \leq \epsilon_{n} \frac{K_{\alpha}}{n-\left[\epsilon_{n}\right]+1}\|x\|, \quad x \in \mathfrak{X}, \quad n \in \mathbb{N} .
$$

As a consequence, (2.8) and (4.5) yield instead of (2.3) (or (1.1)) the operator-norm estimate:

$$
\left\|C^{n}-e^{n(C-\mathbb{1})}\right\| \leq \frac{2}{n^{2 \delta}}+\epsilon_{n} \frac{K_{\alpha}}{n-\left[\epsilon_{n}\right]+1}, n \in \mathbb{N} .
$$


Let $n_{0} \in \mathbb{N}$ satisfies inequality: $2\left(\epsilon_{n_{0}}-1\right) \leq n_{0}$. Then (4.6) gives

$$
\left\|C^{n}-e^{n(C-1)}\right\| \leq \frac{2}{n^{2 \delta}}+\frac{2 K_{\alpha}}{n^{1 / 2-\delta}}, n>n_{0} .
$$

The estimate $M_{\alpha} / n^{1 / 3}$ of the Theorem 4.3 results from the optimal choice of the value: $\delta=1 / 6$, in (4.7).

Similar to $(\sqrt[3]{n})$-Lemma, the $(\sqrt[3]{n})^{-1}$-Theorem is the first step in developing the operator-norm approximation formula à la Chernoff. To this end one needs an operator-norm analogue of Theorem 2.2. Since the last includes the Trotter-Neveu-Kato strong convergence theorem, we need the operator-norm extension of this assertion for quasi-sectorial contractions.

Proposition 4.4. [7] Let $\{X(s)\}_{s>0}$ be a family of m-sectorial operators in a Hilbert space $\mathfrak{H}$ such that for some $0<\alpha<\pi / 2$ and any $s>0$ the numerical range $W(X(s)) \subseteq S_{\alpha}$. Let $X_{0}$ be an $m$-sectorial operator defined in a closed subspace $\mathfrak{H}_{0} \subseteq \mathfrak{H}$, with $W\left(X_{0}\right) \subseteq S_{\alpha}$. Then the two following assertions are equivalent:

$$
\begin{aligned}
& \lim _{s \rightarrow+0}\left\|(\zeta \mathbb{1}+X(s))^{-1}-\left(\zeta \mathbb{1}+X_{0}\right)^{-1} P_{0}\right\|=0, \text { for } \zeta \in S_{\pi-\alpha}, \\
& \lim _{s \rightarrow+0}\left\|e^{-t X(s)}-e^{-t X_{0}} P_{0}\right\|=0, \text { for } t>0 .
\end{aligned}
$$

Here $P_{0}$ denotes the orthogonal projection from $\mathfrak{H}$ onto $\mathfrak{H}_{0}$ and $S_{\alpha}=\{z \in \mathbb{C}:|\arg (z)| \leq \alpha\}$ is a sector in complex plane $\mathbb{C}$ with semi-angle $\alpha$ and vertex at $z=0$.

Now $(\sqrt[3]{n})^{-1}$-Theorem 4.3 and Proposition 4.4 yield a desired generalisation of the operator-norm approximation formula:

Proposition 4.5. [7] Let $\{\Phi(s)\}_{s \geq 0}$ be a family of uniformly quasi-sectorial contractions on a Hilbert space $\mathfrak{H}$, i.e. such that there exists $0 \leq \alpha<\pi / 2$ and $W(\Phi(s)) \subseteq D_{\alpha}$, for all $s \geq 0$. Let

$$
X(s):=(\mathbb{1}-\Phi(s)) / s,
$$

and let $X_{0}$ be a closed operator with non-empty resolvent set, defined in a subspace $\mathfrak{H}_{0} \subseteq \mathfrak{H}$. Then, the family $\left.\{X(s)\}\right\}_{s>0}$ converges, when $s \rightarrow+0$, in the uniform resolvent sense to the operator $X_{0}$ if and only if

$$
\lim _{n \rightarrow \infty}\left\|\Phi(t / n)^{n}-e^{-t X_{0}} P_{0}\right\|=0, \text { for } t>0 \text {. }
$$

Here, $P_{0}$ denotes the orthogonal projection onto the subspace $\mathfrak{H}_{0}$.

Let $A$ be an $m$-sectorial operator with semi-angle $0<\alpha<\pi / 2$ and with vertex at $z=0$, which means that numerical range $W(A) \subseteq S_{\alpha}=\{z \in \mathbb{C}:|\arg (z)| \leq \alpha\}$. Then, $\left\{\Phi(t):=(\mathbb{1}+t A)^{-1}\right\}_{t \geq 0}$ is the family of quasisectorial contractions, i.e., $W(\Phi(t)) \subseteq D_{\alpha}$. Let $X(s):=(\mathbb{1}-\Phi(s)) / s, s>0$, and $X_{0}:=A$. Then, $X(s)$ converges when $s \rightarrow+0$, to $X_{0}$ in the uniform resolvent sense with the asymptotic

$$
\left\|(\zeta \mathbb{1}+X(s))^{-1}-\left(\zeta \mathbb{1}+X_{0}\right)^{-1}\right\|=s\left\|\frac{A}{\zeta \mathbb{1}+A+\zeta s A} \cdot \frac{A}{\zeta \mathbb{1}+A}\right\|=O(s),
$$

for any $\zeta \in S_{\pi-\alpha}$, since we have the estimate:

$$
\left\|\frac{A}{\zeta \mathbb{1}+A+\zeta s A} \cdot \frac{A}{\zeta \mathbb{1}+A}\right\| \leq\left(1+\frac{|\zeta|}{\operatorname{dist}\left(\zeta(1+s \zeta)^{-1},-S_{\alpha}\right)}\right)\left(1+\frac{|\zeta|}{\operatorname{dist}\left(\zeta,-S_{\alpha}\right)}\right) .
$$

Therefore, the family $\{\Phi(t)\}_{t \geq 0}$ satisfies the conditions of Proposition 4.5. This implies the operator-norm approximation of the exponential function, i.e. the semigroup for $m$-sectorial generator, by the powers of resolvent ( $t$ he Euler approximation formula):

Corollary 4.6. $[8,10]$ If $A$ is an $m$-sectorial operator in a Hilbert space $\mathfrak{H}$, with semi-angle $\alpha \in(0, \pi / 2)$ and with vertex at 0 , then

$$
\left\|(\mathbb{1}+t A / n)^{-n}-e^{-t A}\right\| \leq \frac{L_{\alpha}}{n}, \quad t \in S_{\pi / 2-\alpha}
$$

for $n \in \mathbb{N}$. 


\section{Conclusion}

Summarising we note that for the quasi-sectorial contractions instead of divergent (for $n \rightarrow \infty$ ) Chernoff's estimate (1.1), we find the estimate (4.7) which converges for $n \rightarrow \infty$ to zero in the operator-norm topology. Note that the rate $O\left(1 / n^{1 / 3}\right)$ of this convergence is obtained with help of the Poisson representation and the Tchebychëv inequality in the spirit of the proof of Lemma 2.1, and which is not optimal.

The estimate $M / n^{1 / 3}$ in the $(\sqrt[3]{n})^{-1}$-Theorem 4.3 can be improved by a more refined lines of reasoning. For example, by scrutinising our probabilistic arguments one can find a more precise Tchebychëv-type bound for probability of tails. This improves the estimate (4.7) to the rate $O(\sqrt{\ln (n) / n})$, see [11], but again only for quasi-sectorial contractions providing due to Proposition 4.2 the operator-norm contrôl (4.5) of the central part.

On the other hand, a careful analysis of localisation the numerical range of quasi-sectorial contractions [8, 10], generated in a Hilbert space $\mathfrak{H}$ by $m$-sectorial operators with semi-angle $\alpha \in(0, \pi / 2)$, permits one to uplift the operatornorm estimate in Corollary 4.6 to the ultimate optimal $\alpha$-dependent rate $O(1 / n)$, [10, Theorem 4.1].

We note that with help of the spectral representation, one can easily obtain in (4.7) the optimal rate $O(1 / n)$ of the operator-norm convergence for self-adjoint contractions $C$. This is a particular case of the quasi-sectorial contraction for $\alpha=0$, cf. [7, Remark 3.2]. This also concerns the optimal rate of convergence $O(1 / n)$ for the self-adjoint Euler approximation formula (4.10) for $A=A^{*} \geq 0$, which is $m$ (sectorial operator for $\alpha=0$.

\section{References}

[1] Möbus T., Rouzé C. Optimal convergence rate in the quantum Zeno effect for open quantum systems in infinite dimensions. arXiv:2111.13911v2 [quant-ph], 2021, 1-27.

[2] Zagrebnov V.A. Comments on the Chernoff $\sqrt{n}$-lemma. In: Functional Analysis and Operator Theory for Quantum Physics (The Pavel Exner Anniversary Volume), European Mathematical Society, Zürich, 2017, P. 565-573.

[3] Chernoff P.R. Product formulas, nonlinear semigroups and addition of un- bounded operators. Mem. Amer. Math. Soc., 1974, 140, P. 1-121.

[4] Engel K.-J., Nagel R. One-parameter Semigroups for Linear Evolution Equations. Springer-Verlag, Berlin, 2000.

[5] Davies E.B. One-parameter Semigroups. Academic Press, London, 1980.

[6] Kato T. Perturbation Theory for Linear Operators. (Corrected Printing of the Second Edition). Springer-Verlag, Berlin Heidelberg, 1995.

[7] Cachia V., Zagrebnov V.A. Operator-Norm Approximation of Semigroups by Quasi-sectorial Contractions. J. Fuct. Anal., 2001 , 180, P. $176-194$.

[8] Zagrebnov V.A. Quasi-sectorial contractions. J. Funct. Anal., 2008, 254, P. 2503-2511.

[9] Ritt R.K. A condition that $\lim _{n \rightarrow \infty} T^{n}=0$. Proc. Amer. Math. Soc., 1953, 4, P. 898-899.

[10] ArlinskiiYu., Zagrebnov V. Numerical range and quasi-sectorial contractions. J. Math. Anal. Appl., 2010,366, P.33-43.

[11] Paulauskas V. On operator-norm approximation of some semigroups by quasi-sectorial operators. J. Funct. Anal., 2004, 207, P. 58-67.

Submitted 7 January 2022; accepted 9 January 2022

Information about the authors:

V.A. Zagrebnov - Institut de Mathématiques de Marseille - AMU CMI - Technopôle Château-Gombert 39 rue F. Joliot Curie, 13453 Marseille, France; Valentin.Zagrebnov@univ-amu.fr

Conflict of interest: the author declare no conflict of interest. 